\title{
Whole-body diffusion-weighted MRI of normal lymph nodes: prospective apparent diffusion coefficient histogram and nodal distribution analysis in a healthy cohort
}

\author{
Ricardo Donners ${ }^{1 *+} \mathbb{D}$, Raphael Shih Zhu Yiin ${ }^{2+}$, Matthew Blackledge ${ }^{3}$ and Dow-Mu Koh ${ }^{4}$
}

\begin{abstract}
Background: Whole body DWI (WB-DWI) enables the identification of lymph nodes for disease evaluation. However, quantitative data of benign lymph nodes across the body are lacking to allow meaningful comparison of diseased states. We evaluated apparent diffusion coefficient (ADC) histogram parameters of all visible lymph nodes in healthy volunteers on WB-DWI and compared differences in nodal ADC values between anatomical regions.

Methods: WB-DWI was performed on a 1.5 T MR system in 20 healthy volunteers (7 female, 13 male, mean age 35 years). The b900 images were evaluated by two radiologists and all visible nodes from the neck to groin areas were segmented and individual nodal median ADC recorded. All segmented nodes in a patient were summated to generate the total nodal volume. Descriptors of the global ADC histogram, derived from individual node median ADCs, including mean, median, skewness and kurtosis were obtained for the global volume and each nodal region per patient. ADC values between nodal regions were compared using one-way ANOVA with Bonferroni post hoc tests and a $p$-value $\leq 0.05$ was deemed statistically significant.

Results: One thousand sixty-seven lymph nodes were analyzed. The global mean and median ADC of all lymph nodes were $1.12 \pm 0.27\left(10^{-3} \mathrm{~mm}^{2} / \mathrm{s}\right)$ and $1.09\left(10^{-3} \mathrm{~mm}^{2} / \mathrm{s}\right)$. The average median ADC skewness was $0.25 \pm 0.02$ and average median ADC kurtosis was $0.34 \pm 0.04$. The ADC values of intrathoracic, portal and retroperitoneal nodes were significantly higher $\left(1.53 \times 10^{-3}, 1.75 \times 10^{-3}\right.$ and $1.58 \times 10^{-3} \mathrm{~mm}^{2} / \mathrm{s}$ respectively $)$ than in other regions. Intrathoracic, portal and mesenteric nodes were relatively uncommon, accounting for only $3 \%$ of the total nodes segmented.

Conclusions: The global mean and median ADC of all lymph nodes were $1.12 \pm 0.27\left(10^{-3} \mathrm{~mm}^{2} / \mathrm{s}\right)$ and $1.09\left(10^{-3}\right.$ $\mathrm{mm}^{2} / \mathrm{s}$ ). Intrathoracic, portal and retroperitoneal nodes display significantly higher ADCs. Normal intrathoracic, portal and mesenteric nodes are infrequently visualized on WB-DWI of healthy individuals.
\end{abstract}

Trial registration: Royal Marsden Hospital committee for clinical research registration number 09/H0801/86, 19.10.2009.

Keywords: Whole-body MRI, Diffusion-weighted MRI, Lymph nodes, Histogram

\footnotetext{
* Correspondence: ricardo.donners@rmh.nhs.uk

${ }^{\dagger}$ Ricardo Donners and Raphael Shih Zhu Yiin are co-first authors.

'Department of Diagnostic Radiolog, The Royal Marsden NHS Foundation

Trust, Downs Road, Sutton, London, Surrey SM2 5PT, UK

Full list of author information is available at the end of the article
}

C C The Author(s). 2021 Open Access This article is licensed under a Creative Commons Attribution 4.0 International License, which permits use, sharing, adaptation, distribution and reproduction in any medium or format, as long as you give appropriate credit to the original author(s) and the source, provide a link to the Creative Commons licence, and indicate if changes were made. The images or other third party material in this article are included in the article's Creative Commons licence, unless indicated otherwise in a credit line to the material. If material is not included in the article's Creative Commons licence and your intended use is not permitted by statutory regulation or exceeds the permitted use, you will need to obtain permission directly from the copyright holder. To view a copy of this licence, visit http://creativecommons.org/licenses/by/4.0/. The Creative Commons Public Domain Dedication waiver (http://creativecommons.org/publicdomain/zero/1.0/) applies to the data made available in this article, unless otherwise stated in a credit line to the data. 


\section{Background}

Lymph nodes are commonly affected by malignant disease and their involvement impacts patient management [1]. Consequently, early detection and accurate staging of malignant lymph nodes through imaging is of paramount clinical importance. Their evaluation by conventional CT or MRI relies on size thresholds and subjective, non-quantitative assessment of morphologic features to discriminate between diseased and normal states. This method was shown to have limited diagnostic accuracy [2].

Whole body diffusion weighted MRI (WB-DWI) has emerged as a functional imaging technique, which can aid lymph node characterization by providing information on tissue characteristics over a large field of view within reasonable acquisition times - properties, which make it a desirable staging and screening technique [3, 4]. WB-DWI was deemed an alternative to established ${ }^{18}$ FDG PET/CT for lymphoma staging [5]. DWI detects microstructural and cellular alterations in malignant versus benign lymph nodes and can be quantified by calculation of the apparent diffusion coefficient (ADC). The latter was shown to inversely correlate with cellularity, with good measurement repeatability, allowing for tissue characterization [6-9]. Several regional and WB-DWI studies have shown lower ADC values in malignant compared to non-diseased nodes, suggesting the use of ADC as a biomarker for lymph node assessment [1018]. However, these studies report overlap between malignant and benign states and as a consequence a universal, consistent ADC threshold that can be applied across different cancers to accurately identify malignancy is lacking. One major contributor to this problem is that the published literature on ADC values of benign nodes were derived from patients with pre-existing malignancies. Data on ADC values of normal lymph nodes of a healthy population are lacking. Additionally, there is paucity of data with regards to how nodal ADC values may vary across anatomical sites. Establishment of nodal ADC ranges throughout the body and regional differences in unequivocally normal lymph nodes may facilitate identification of diseased states. Consequently, the purpose of this study is to determine the WB-DWI derived ADC values of normal lymph nodes of healthy individuals and compare them between anatomical regions to establish healthy, nodal ADC ranges.

\section{Methods}

\section{Study design and study population}

Approval for this prospective, non-randomized study performed at a single institution was obtained from the local institutional research and ethics committee. Twenty healthy adult volunteers were recruited and written informed consent was obtained. Exclusion criteria were previous surgery, known or prior malignancy, acute inflammation or infection within 4 weeks prior to scanning and current use of short- or long-term medication.

\section{Whole-body MR imaging protocol}

Free-breathing axial single-shot, spin-echo, echo planar imaging WB-DWI was performed on a 1.5 T MRI scanner (MAGNETOM Avanto, Siemens Healthineers, Erlangen, Germany) using the following imaging parameters: Matrix $=150 \times 144$, Partial Fourier $=6 / 8$, $\mathrm{TE}=64.8 \mathrm{~ms}, \quad \mathrm{TR}=14.6 \mathrm{~s}, \quad$ Receiver Bandwidth $=1961$ $\mathrm{Hz} / \mathrm{px}, \mathrm{b}$-values $=50$ and $900 \mathrm{~s} / \mathrm{mm}^{2}$, three scan trace weighted diffusion encoding, STIR fat suppression $(\mathrm{TI}=$ $180 \mathrm{~ms}$ ), Slice Thickness $=5 \mathrm{~mm}$, Field of View $=400 \times$ $390 \mathrm{~mm}^{2}$. GRAPPA was applied to reduce distortion along the phase-encoding direction $(R=2)$. The number of signal averages was four. Images were acquired from vertex to midthigh using four consecutive imaging stations comprising of 50 slices each. The acquisition time was approximately $24 \mathrm{~min}$. To facilitate anatomical localization of lymph nodes, breath-hold axial T1weighted MRI, matched to the same imaging field of view and slice thickness of WB-DWI were acquired using the following imaging parameters: T1/FL2D, matrix $=256 \times 105$, slice thickness $=5 \mathrm{~mm}$, spacing $=5$ $\mathrm{mm}, \mathrm{TR}=386 \mathrm{~ms}, \mathrm{TE}=4.8 \mathrm{~ms}$, flip angle $=70^{\circ}$, number of averages $=1$.

\section{Image interpretation and processing}

The b900 DW images were reviewed by two radiologists with six and eight years of experience in MRI interpretation, respectively. Open-access software (OsiriX version 56, PixmeoSARL Bernex, Switzerland) was used for analyses and post-processing. Facilitated by the anatomical T1-weighted sequences, all lymph nodes were identified on high b-value images as discrete ovoid structures demonstrating high signal intensity and appeared intermediate to low signal intensity, some with a fatty, hilum on the corresponding T1-weighted images. A volume of interest (VOIs) was generated, encompassing an individual node on b900 images, using a semi-automated segmentation software within OsiriX employing a "GrowCUT" algorithm [19]. This was repeated for every lymph node visible on b900 images. When necessary, VOIs were manually adjusted to the nodal parenchyma. All nodal VOIs of a volunteer were transferred onto the corresponding ADC maps. For each lymph node, the nodal volume $\left(\mathrm{ml}\right.$ ) and median ADC (in $10^{-3} \mathrm{~mm}^{2} / \mathrm{s}$ ) were recorded.

In each volunteer, all individual nodal volumes were summated to create the global nodal volume $(\mathrm{ml})$. From this global volume the global ADC (gADC) histogram, based on individual node median ADC values was 
derived. Histogram descriptors including mean, median, skewness and kurtosis were recorded.

Additionally, individual nodal volumes were summarized per anatomical site to create regional nodal volumes $(\mathrm{ml})$. From these regional volumes, mean and median of the included individual median nodal ADCs were recorded. The workflow is visualized in Fig. 1.

\section{Lymph node regions}

The lymph nodes were grouped according to ten anatomical sites: occipital, cervical, axillary, intra-thoracic, portal, mesenteric, retroperitoneal, pelvic, inguinal and subcutaneous regions.
The occipital nodes extend from the occiput to the level of the skull base [20]. The cervical region reaches from the skull base to the supra-clavicular region, with reference made to the imaging based nodal classification for metastatic neck adenopathy [21]. The axillary region is defined as the anatomical axillary triangle [22], which only consist of level 1 axillary lymph nodes in our study. The level 2 and 3 axillary lymph nodes (including infraclavicular nodes) were not encountered and hence not evaluated. The intrathoracic group comprises the mediastinal nodal stations defined by the International Association for the Study of Lung cancer (IASLC), including the internal mammary nodes and excluding the supraclavicular nodes [23]. The portal group summarises

\section{Lymph node segmentation}
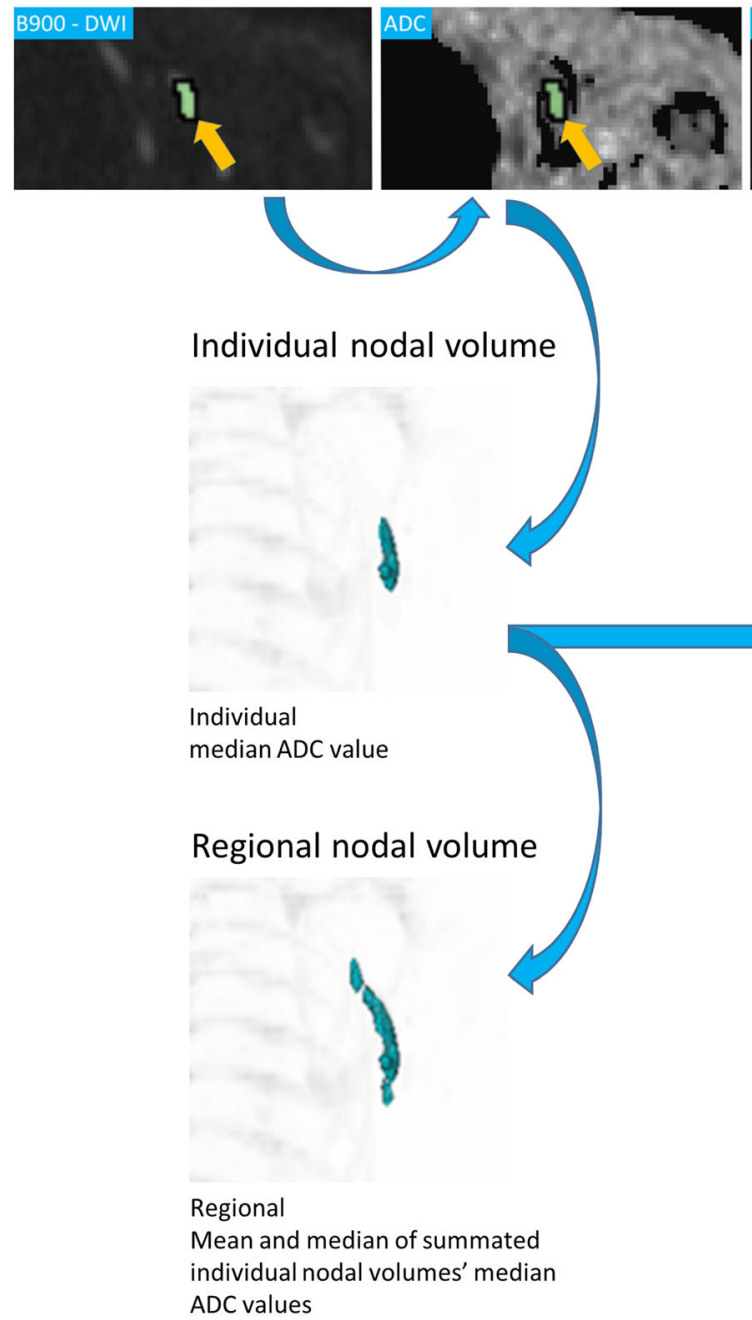

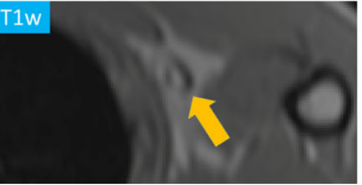

Global nodal volume

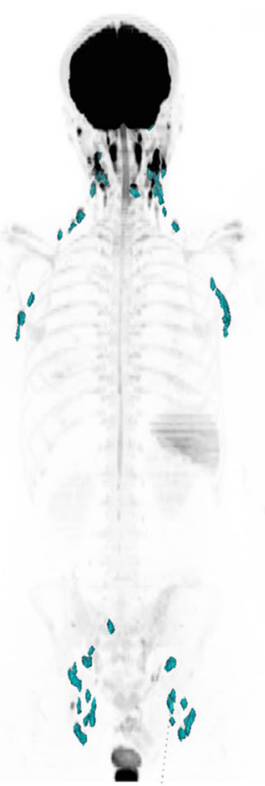

Global

Mean, median, skewness, kurtosis of summated individual nodal volumes' median ADC values

Fig. 1 Workflow of nodal volume segmentation and Apparent Diffusion Coefficient (ADC) measurement in a 30-year-old male. A left axillary lymph node is identified on anatomical T1 weighted (T1w) and b900 DWI (arrows) and segmented (green) on b900 images. The resulting volume of interest (VOI) was copied onto the ADC map and individual nodal median ADC derived. Individual nodal volumes were summated to regional (axillary group) and global nodal volumes. Individual nodal median ADC distribution descriptors within these summated volumes (mean, median, skewness, kurtosis) were derived 
nodes along the portal vein, with the inferior limit set at the spleno-portal venous axis, while the mesenteric group is composed of nodes within the bowel mesentery. The retroperitoneal group includes nodes within the retroperitoneal space. The upper limit is set just inferior to the aortic hiatus and the lower limit at the iliac bifurcation. The pelvic group comprises nodes along the anatomic boundaries of the iliac vessels and pelvic sidewalls. The inguinal group consists of nodes immediately above and below the inguinal ligament, as well as deep nodes medial to the femoral vein. Subcutaneous nodes are superficial nodes that are identified within the subcutaneous tissue anywhere in the body and not included in any one of the defined groups.

\section{Statistical analysis}

Statistical analyses were performed using commercially available software (IBM SPSS Statistics Version 22, IBM Corp. Armonk, and SAS JMP, SAS Institute Inc.). The median ADC parameters were compared between nodal regions utilizing one-way analysis of variance (ANOVA) testing with Bonferroni post hoc tests. Normal distribution was assessed using the Shapiro-Wilk-test. A $p$-value $\leq 0.05$ was taken to be statistically significant. The distributions of median ADC and nodal volume are approximated by kernel density estimation using a Gaussian kernel and a bandwidth approximated by Silverman's rule of thumb [24]. Pearson coefficients were calculated to assess correlation between volunteer age and global ADC.

\section{Results}

Seven women and 13 men were recruited, with a mean age of 34 years (range 21-59 years). In total 1067 nodes were identified and segmented.

\section{Nodal distribution}

Lymph node distributions on WB-DWI were observed as follows: occipital (6.6\%), cervical (32.2\%), axillary (18.4\%), intrathoracic $(0.9 \%)$, mesenteric $(1.2 \%)$, portal (1\%), retroperitoneal (8.9\%), pelvic (6\%), inguinal (23.9\%) and subcutaneous (0.7\%). Figure 2 shows the lymph node volumes as identified on a WB-DWI 3D Maximum intensity projection (MIP) of a 30-year-old healthy male.

\section{Nodal volume}

The mean \pm standard deviation of single lymph node volumes was $0.48 \pm 0.44 \mathrm{ml}$, with a median volume of 0.34 $\mathrm{ml}$ and modal volume of $0.12 \mathrm{ml}$, with values ranging between 0.05 and $2.93 \mathrm{ml}$. Figure 3 shows the distribution histogram for all nodal volumes across the study cohort.

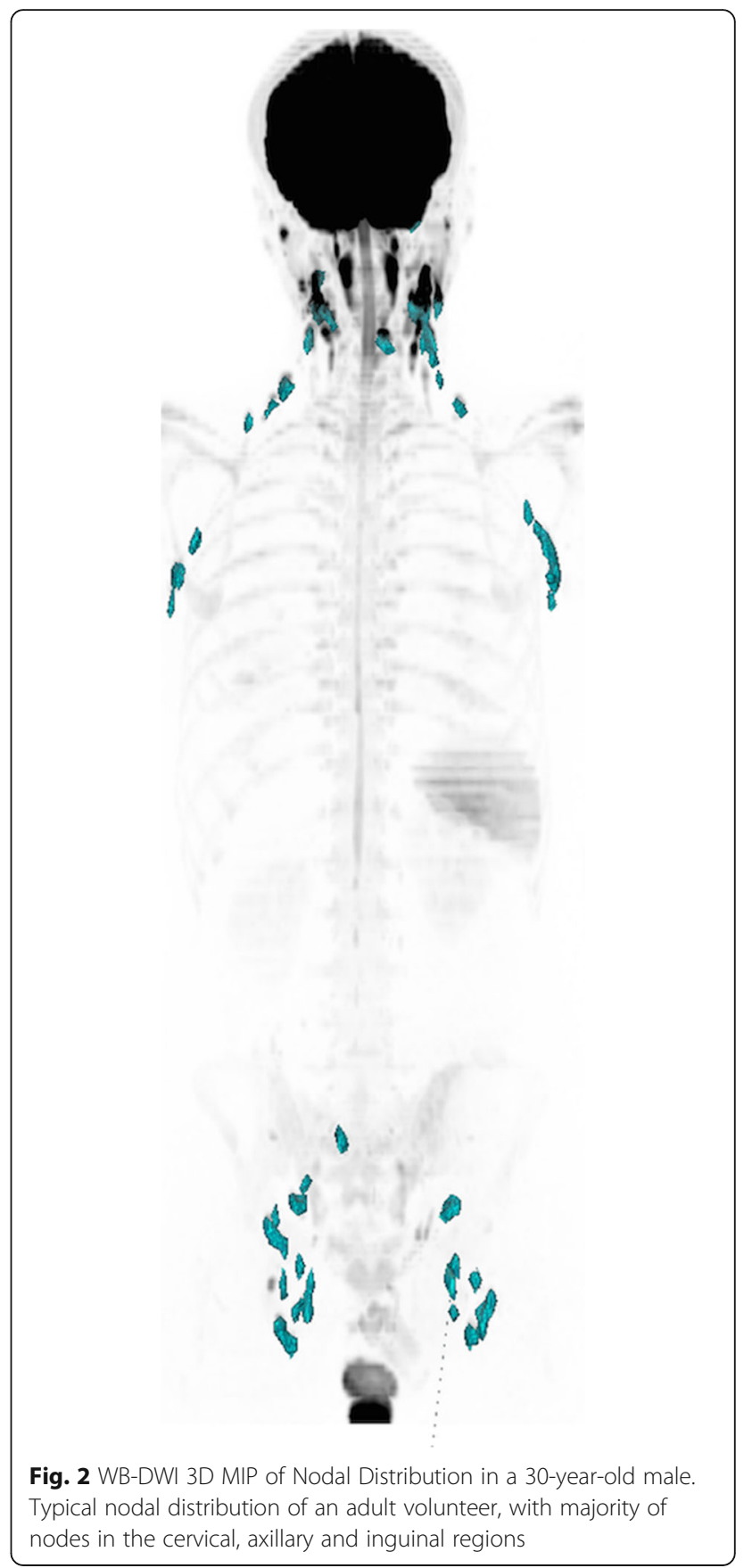

ADC histogram parameters of the total measured lymph node volume

The median global ADC values per volunteer are shown in Table 1. The global mean and median of individual nodal median ADC values across all 20 volunteers were $1.12 \pm 0.27\left(10^{-3} \mathrm{~mm}^{2} / \mathrm{s}\right)$ and $1.09\left(10^{-3} \mathrm{~mm}^{2} / \mathrm{s}\right)$ respectively. Figure 4 shows the histogram distribution curve for the nodal median ADC values of all normal lymph nodes across the study cohort. The average skewness of nodal median ADC was $0.25 \pm 0.02$; and the kurtosis was $0.34 \pm 0.04$. No differences between genders were 


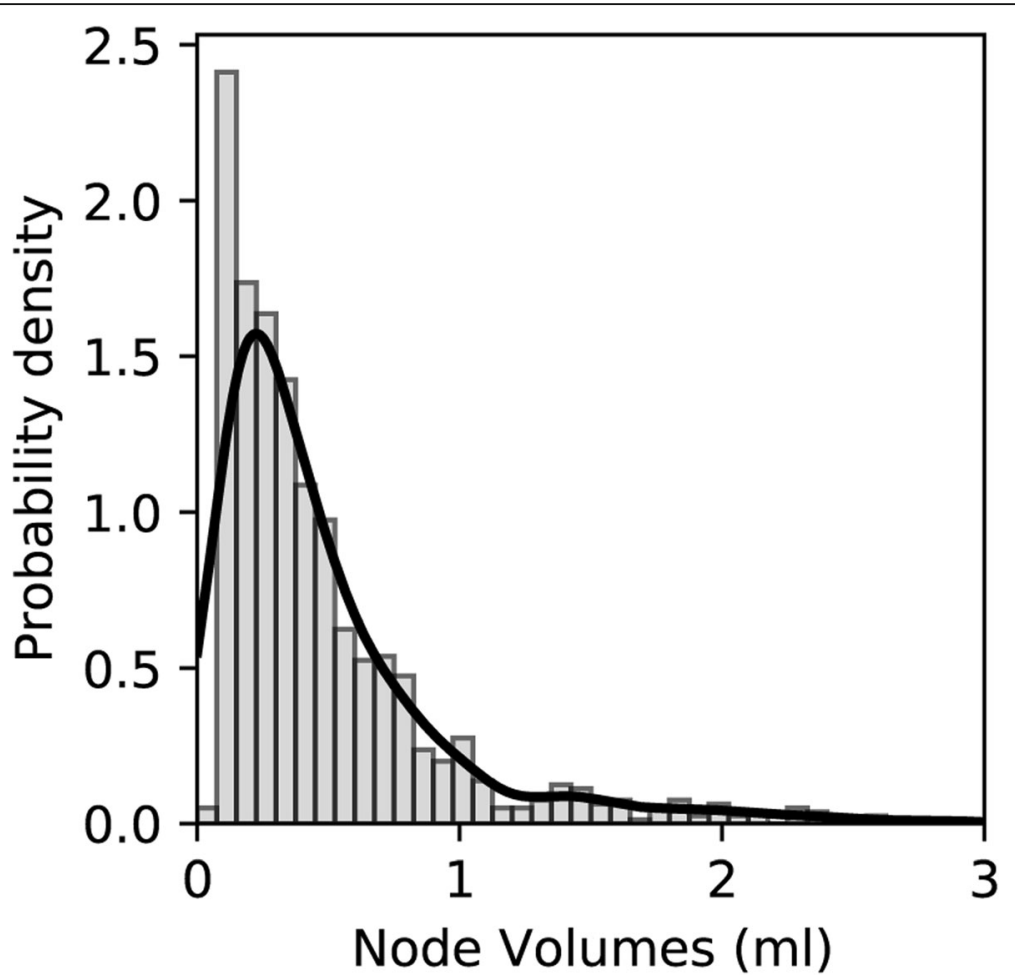

Fig. 3 Histogram and kernel density estimation (solid line) showing the distribution of nodal volumes across the entire study cohort including all nodal regions of all participants

Table 1 Data table showing the nodal frequency, mean nodal volume $(\mathrm{ml})$ and median global ADC values $\left(10^{-3} \mathrm{~mm}^{2} / \mathrm{s}\right)$ of the 20 volunteers. SD = standard deviation

\begin{tabular}{|c|c|c|c|c|}
\hline Volunteers & Number of nodes & Mean Nodal Volume in $\mathrm{ml}$ & SD & Median ADC $\times 10^{3} \mathrm{~mm} / \mathrm{s}^{2}$ \\
\hline 1 & 62 & 0.50 & 0.45 & 0.99 \\
\hline 2 & 51 & 0.50 & 0.37 & 1.19 \\
\hline 3 & 26 & 0.54 & 0.58 & 1.16 \\
\hline 4 & 30 & 0.57 & 0.48 & 1.16 \\
\hline 5 & 40 & 0.48 & 0.52 & 1.00 \\
\hline 6 & 76 & 0.77 & 0.59 & 0.91 \\
\hline 7 & 70 & 0.51 & 0.34 & 1.25 \\
\hline 8 & 44 & 0.47 & 0.39 & 1.16 \\
\hline 9 & 69 & 0.37 & 0.32 & 1.14 \\
\hline 10 & 60 & 0.65 & 0.70 & 1.15 \\
\hline 11 & 57 & 0.51 & 0.55 & 1.26 \\
\hline 12 & 62 & 0.48 & 0.31 & 1.03 \\
\hline 13 & 41 & 0.70 & 0.67 & 1.22 \\
\hline 14 & 47 & 0.33 & 0.19 & 1.03 \\
\hline 15 & 61 & 0.46 & 0.40 & 1.00 \\
\hline 16 & 54 & 0.74 & 0.57 & 1.02 \\
\hline 17 & 55 & 0.33 & 0.23 & 1.00 \\
\hline 18 & 57 & 0.57 & 0.41 & 0.90 \\
\hline 19 & 54 & 0.64 & 0.69 & 1.14 \\
\hline 20 & 51 & 1.17 & 0.77 & 0.92 \\
\hline
\end{tabular}




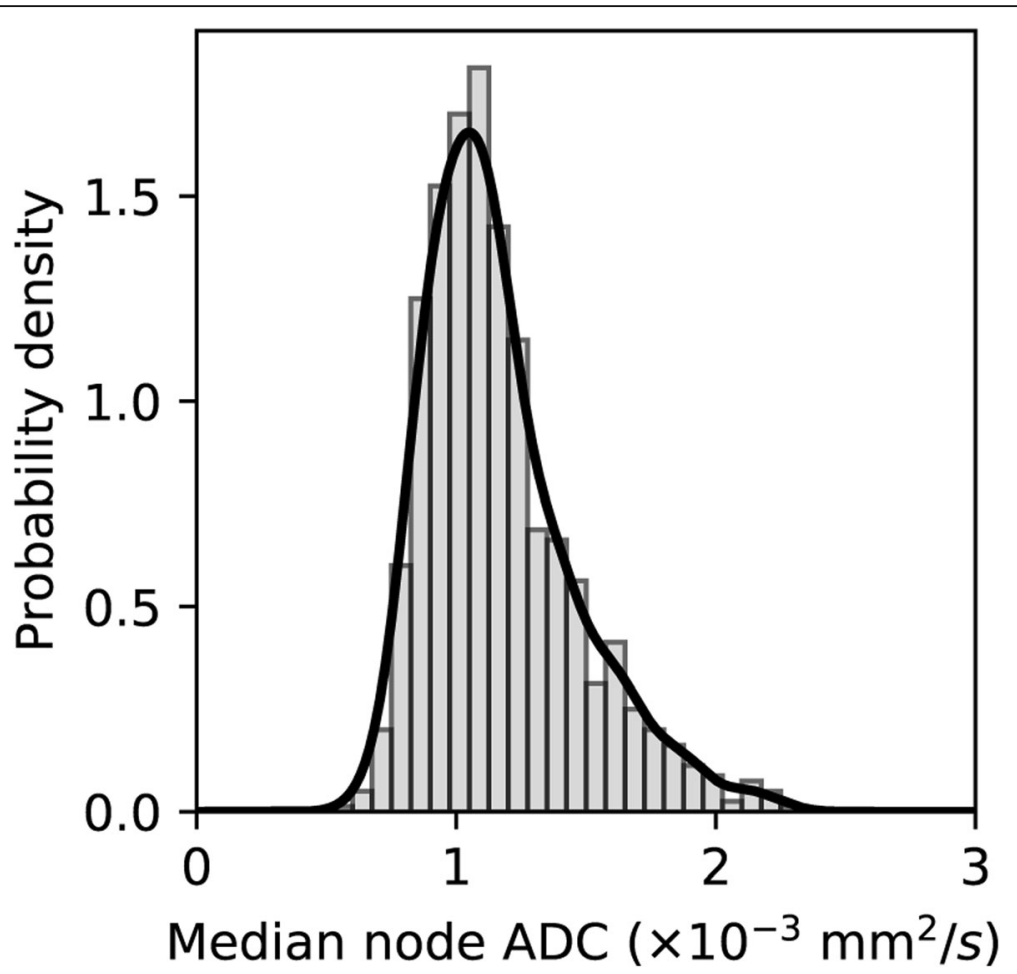

Fig. 4 Histogram and kernel density estimation (solid line) showing the distribution of median ADC values of all normal lymph nodes across the entire study cohort including all nodal regions of all participants

observed $(p>0.05)$. Pearson correlation showed no significant correlation between patient age and global mean/median nodal median ADC $(p>0.348$, Pearson correlation $=-0.222$ ).

\section{Comparison across nodal regions}

The nodal frequency, mean nodal volumes and median ADC values per anatomical region across the study cohort are shown in Table 2.

Figure 5 shows the box-and-whiskers plot of nodal median ADC values according to the nodal regions defined in our study.

The regional lymph node median $\mathrm{ADC}$ values ranged from 0.98 to $1.18\left(10^{-3} \mathrm{~mm}^{2} / \mathrm{s}\right)$ for the cervical, inguinal, axillary, occipital, mesenteric, pelvic and subcutaneous nodal groups, comprising $88 \%$ of all included lymph nodes. Compared with all other nodal regions, retroperitoneal and intrathoracic nodes showed significantly higher nodal median ADC $\left(1.61 \times 10^{-3}\right.$ and $1.75 \times 10^{-3} \mathrm{~mm}^{2} / \mathrm{s}$ respectively, $p<0.001)$. Portal nodes also showed significantly higher median ADC $\left(1.58 \times 10^{-3} \mathrm{~mm}^{2} / \mathrm{s}\right)$ when compared with the regions of high nodal frequency, but not when compared with the mesenteric region $(p=0.173)$.

Among the three most common nodal groups (cervical, axillary and inguinal), each containing more than 190 evaluated nodes in this study, cervical nodes displayed higher median ADC values than axillary or inguinal nodes $(\mathrm{p}<0.001)$. By contrast, intrathoracic, portal and mesenteric nodes were relatively uncommon, accounting for only $3 \%$ of the total nodes evaluated in our study.

\section{Discussion}

The early detection and accurate staging of malignant nodal disease is important for patient management, but conventional $\mathrm{CT}$ and MRI have limited diagnostic performance. Quantitative WB-DWI may improve nodal assessment, but there is paucity of data concerning normal lymph node ADCs.

This study uniquely describes ADC histogram characteristics derived from more than 1000 lymph node volumes of a healthy adult population. The global mean and median ADC of all lymph nodes were $1.12 \pm 0.27$ and $1.09\left(10^{-3} \mathrm{~mm}^{2} / \mathrm{s}\right)$, respectively. Most lymph nodes were identified in the cervical, inguinal and axillary region. In prior regional DWI studies, including only the cervical, axillary and inguinal lymph nodes of 13, 16 and 20 healthy volunteers, respectively, the mean ADC of normal lymph nodes ranged between 0.87 and 1.18 $\left(10^{-3} \mathrm{~mm}^{2} / \mathrm{s}\right)$ [11, 16, 25]. In another study of pelvic nodes in eight healthy volunteers, the mean ADC was $1.28\left(\times 10^{-3} \mathrm{~mm}^{2} / \mathrm{s}\right)$ [26]. Previous authors noted that normal lymph nodes in the chest and abdomen were poorly depicted on DWI and therefore excluded from 
Table 2 Data table showing the nodal frequency, mean nodal volume $(\mathrm{ml})$ and median ADC values $\left(10^{-3} \mathrm{~mm}^{2} / \mathrm{s}\right)$ per nodal region. $\mathrm{SD}=$ standard deviation, $95 \%-\mathrm{Cl}=$ confidence interval

\begin{tabular}{llllll}
\hline Nodal region & Node count & Mean nodal volume in $\mathrm{ml}$ & SD & Median ADC x10 $\mathrm{mm}^{3} \mathrm{~s}^{2}$ & $95 \% \mathrm{Cl}$ \\
\hline All nodes & 1067 & 0.48 & 0.45 & 1.11 & $1.09-1.12$ \\
Cervical & 344 & 0.36 & 0.15 & 1.14 & $1.10-1.16$ \\
Inguinal & 255 & 0.68 & 0.54 & 0.99 & $0.97-1.01$ \\
Axilla & 196 & 0.61 & 0.54 & 1.06 & $1.03-1.08$ \\
Retroperitoneal & 95 & 0.32 & 0.25 & 1.61 & $1.53-1.67$ \\
Pelvic & 65 & 0.50 & 0.41 & 1.17 & $1.12-1.29$ \\
Occipital & 70 & 0.32 & 0.21 & 1.14 & $1.12-1.21$ \\
Mesenteric & 13 & 0.46 & 0.25 & 1.18 & $1.08-1.55$ \\
Portal & 11 & 0.39 & 0.26 & 1.58 & $1.33-1.66$ \\
Intrathoracic & 10 & 0.51 & 0.57 & 1.75 & $1.40-1.92$ \\
Subcutaneous & 8 & 0.21 & 0.07 & 0.97 & $0.84-1.41$ \\
\end{tabular}

analyses $[11,16]$. In our study, intrathoracic and some abdominal nodes are also less frequently encountered. The sum of intrathoracic, portal and mesenteric nodes represented only $3 \%$ of the total nodes segmented in our study. This may be related to artefacts caused by cardiac and respiratory motion, causing loss of signal and ultimately low visibility on DWI. The significantly higher $\mathrm{ADC}$ values and ranges displayed by intrathoracic, portal and retroperitoneal nodes might be attributed to motion of adjacent organs, sampling bias and gradient nonlinearity. DWI signal decay caused by the random movement of water molecules can be accentuated by nonlinear cardiac or bowel movement, resulting in greater signal decay and consequently overestimation of ADC. As a consequence, nodal ADC of thoracic and some abdominal regions need to be interpreted with caution.

The whole body protocol used in this study follows general clinical recommendations in the choice of b-

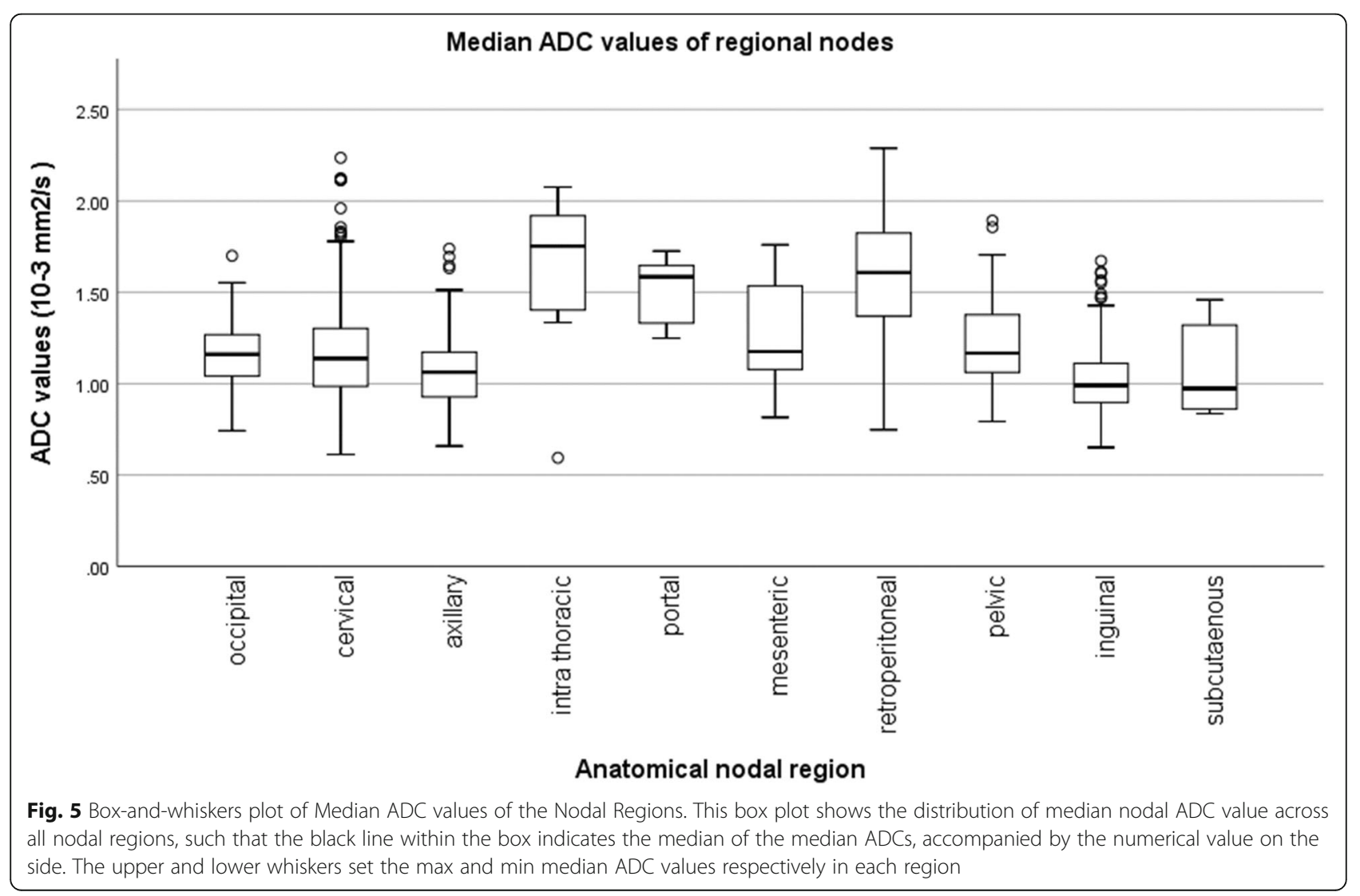


values, facilitating comparability [27, 28]. The observed measurements lie within mean $\mathrm{ADC}$ ranges of previous WB-MRI studies with values of nodes deemed normal on imaging ranging between 1.00 and $1.37 \times 10^{-3} \mathrm{~mm}^{2} / \mathrm{s}$ $[11,13,29]$. Additionally, ADC values were reported to have inter-scanner variability of $14 \%$, accentuated in small volume measurements such as lymph nodes [30]. This may also contribute to measurement differences between centers. It needs to be noted however, that these studies derived "normal "nodal ADCs from patients with underlying malignancy. Hence, these controls may have been subject to reactive changes secondary to treatment or inflammation, which could also affect nodal diffusion characteristics. In contrast, potential confounders in our healthy cohort have been accounted for in the exclusion criteria of this study.

Evaluating more than 1000 lymph nodes allowed us to define the full ADC histogram characteristics of normal lymph nodes. Histogram parameters such as mean, median, skewness and kurtosis were shown to be potential response biomarkers in various types of cancers and lymphoma [12, 13, 31-36]. Consequently, establishing values for normal, healthy conditions is important to allow comparisons also for baseline examinations. One study identified skewness to be a better discriminator between FDG-PET/CT positive and negative lymph nodes in lymphoma patients than mean ADC [13]. In this study, skewness was derived from individual nodes. In contrast, we derived skewness and kurtosis values from the distribution of median individual node ADC values summarized on a per patient or per nodal group basis. Consequently the findings are not directly comparable. Nevertheless, skewness and kurtosis have shown poor repeatability in WB-DWI, and their application requires critical evaluation [37].

Overall, there is large potential for WB-DWI in assessment of nodal disease across the body, as it allows for generation of functional tissue information within short acquisition times, without radioactivity or contrast application. Contemporary literature suggests no significant alteration of $\mathrm{T} 2$ relaxation times between normal and diseased nodes, thus making signal and ADC changes dependent on diffusion properties [38]. Knowledge of healthy nodal ADC parameters can improve disease evaluation and is especially useful in morphologically unremarkable nodes. DWI assessment of lymph nodes can become more relevant in the future, as acceleration techniques such as simultaneous multislice DWI acquisition [26] and development of machine learning applications may enhance image quality without increasing acquisition time. Machine learning may also allow fast, automated nodal segmentation and identification of normal nodes and potentially diseased states defined on the basis of ADC thresholds.
This study has limitations, mostly related to the DWI technique. First, misregistration and partial volume effects may degrade ADC assessment of small nodes, the latter was shown to cause up to $12.8 \%$ decrease in ADC measurement reproducibility [25]. Second, the ADC may vary over the field of view due to gradient non-linearity [39]. Third, images were acquired on a single scanner, hence immediate application of ADC ranges to other scanners is limited. Nevertheless, the protocol and sequence used are common clinical standard and should allow for general approximation. Fourth, the inter- and intra-observer repeatability of the measurements have not been investigated in this study. Finally, the mean age of 35 years of the volunteers is well below the age of the average cancer patient. Nonetheless, in our limited cohort we could find no significant age dependence of nodal ADCs.

\section{Conclusions}

In conclusion, the global mean and median ADC of all lymph nodes were $1.12 \pm 0.27\left(10^{-3} \mathrm{~mm}^{2} / \mathrm{s}\right)$ and 1.09 $\left(10^{-3} \mathrm{~mm}^{2} / \mathrm{s}\right)$. Intrathoracic, portal and retroperitoneal nodes display significantly larger ADCs. Normal intrathoracic, portal and mesenteric nodes are infrequently visualized on WB-DWI of healthy individuals.

\section{Abbreviations \\ ADC: Apparent diffusion coefficient; DWI: Diffusion-weighted MRI; gADC: Global apparent diffusion coefficient; KDE: Kernel density estimate; TE: Echo time; TR: Repetition time; STIR: Short tau inversion recovery; WB: Whole-body}

\section{Acknowledgements}

Not applicable.

\section{Authors' contributions}

RD - Conceptualization; Data curation; Formal analysis; Investigation; Methodology; Project administration; Resources; Software; Validation; Visualization; Roles/Writing - original draft; Writing - review \& editing. RYSZ Conceptualization; Data curation; Formal analysis; Investigation;

Methodology; Project administration; Resources; Software; Validation; Visualization; Roles/Writing - original draft; Writing - review \& editing. MB Conceptualization; Formal analysis; Investigation; Methodology; Project administration; Resources; Supervision; Validation; Visualization; Roles/Writing - original draft; Writing - review \& editing. DMK - Conceptualization; Formal analysis; Investigation; Methodology; Project administration; Resources; Supervision; Validation; Visualization; Roles/Writing - original draft; Writing review \& editing. The author(s) read and approved the final manuscript.

\section{Funding}

This report is independent research funded by the National Institute for Health Research, Grant information: Cancer Research UK and EPSRC to the Cancer Imaging Centre at ICR and RMH, in association with the MRC and Department of Health (England) (C1060/A10334 \& C1060/A16464). Funding to the NIHR Biomedical Research Centre and Clinical Research Facilities at The Royal Marsden and the Institute of Cancer Centre.

The shared first author, Ricardo Donners, received funding from a research grant of the University Basel and a scholarship from the Swiss Society of Radiology (SSR-SGR) and this work was partially generated in the funded time period. The funding organisations had no impact on the design of the study nor in writing this manuscript. The views expressed in this publication are those of the authors and not necessarily those of any funding body, 
including the NHS, the National Institute for Health Research or the Department of Health.

\section{Availability of data and materials}

The datasets analysed during the current study are available from the corresponding author on reasonable request.

\section{Declarations}

\section{Ethics approval and consent to participate}

Approval for this study was obtained from the committee for clinical research of the Royal Marsden Hospital, NHS foundation trust, research registration number 09/H0801/86,

\section{Consent for publication}

Not applicable.

\section{Competing interests}

The authors declare that they have no competing interests.

\section{Author details}

'Department of Diagnostic Radiolog, The Royal Marsden NHS Foundation Trust, Downs Road, Sutton, London, Surrey SM2 5PT, UK. ²Department of Diagnostic Radiology, Changi General Hospital, 2 Simei St 3, Singapore 529889, Singapore. ${ }^{3}$ Institute of Cancer Research, 15 Cotswold Road, Sutton London SM2 5NG, UK. ${ }^{4}$ Department of Diagnostic Radiology, Institute of Cancer Research and The Royal Marsden NHS, Foundation Trust, Downs Road, Sutton, London, Surrey SM2 5PT, UK

\section{Received: 29 March 2021 Accepted: 12 November 2021}

Published online: 27 November 2021

\section{References}

1. Torabi M, Aquino SL, Harisinghani MG. Current concepts in lymph node imaging. J Nucl Med. 2004;45(9):1509-18.

2. Herneth AM, Mayerhoefer M, Schernthaner R, Ba-Ssalamah A, Czerny C, Fruehwald-Pallamar J. Diffusion weighted imaging: lymph nodes. Eur J Radiol. 2010;76(3):398-406

3. Tunariu N, Blackledge M, Messiou C, Petralia G, Padhani A, Curcean S, et al. What's new for clinical whole-body MRI (WB-MRI) in the 21 st century. $\mathrm{Br}$ J Radiol. 2020;93(1115):20200562.

4. Cheson BD. Staging and response assessment in lymphomas: the new Lugano classification. Chin Clin Oncol. 2015;4(1):5.

5. Kharuzhyk S, Zhavrid E, Dziuban A, Sukolinskaja E, Kalenik O. Comparison of whole-body MRI with diffusion-weighted imaging and PET/CT in lymphoma staging. Eur Radiol. 2020;30(7):3915-23.

6. Mao Y, Hedgire S, Harisinghani M. Radiologic Assessment of Lymph Nodes in Oncologic Patients. Current Radiology Reports. 2013;2(2).

7. Le Bihan D. Molecular diffusion nuclear magnetic resonance imaging. Magn Reson Q. 1991;7(1):1-30.

8. Koh DM, Collins DJ. Diffusion-weighted MRI in the body: applications and challenges in oncology. AJR Am J Roentgenol. 2007;188(6):1622-35.

9. Donners R, Blackledge M, Tunariu N, Messiou C, Merkle EM, Koh DM Quantitative whole-body diffusion-weighted MR imaging. Magn Reson Imaging Clin N Am. 2018;26(4):479-94.

10. Fitzpatrick JJ, Ryan MA, Bruzzi JF. Diagnostic accuracy of diffusion-weighted imaging- magnetic resonance imaging compared to positron emission tomography/computed tomography in evaluating and assessing pathological response to treatment in adult patients with lymphoma: A systematic review. J Med Imaging Radiat Oncol. 2018. Mar 25.

11. Kwee TC, Ludwig I, Uiterwaal CS, van Ufford HM, Vermoolen MA, Fijnheer $R$, et al. ADC measurements in the evaluation of lymph nodes in patients with non-Hodgkin lymphoma: feasibility study. MAGMA. 2011;24(1):1-8.

12. Wang YJ, Xu XQ, Hu H, Su GY, Shen J, Shi HB, et al. Histogram analysis of apparent diffusion coefficient maps for the differentiation between lymphoma and metastatic lymph nodes of squamous cell carcinoma in head and neck region. Acta Radiol. 2017;284185117730688.

13. De Paepe KN, De Keyzer F, Wolter P, Bechter O, Dierickx D, Janssens A, et al. Improving lymph node characterization in staging malignant lymphoma using first-order ADC texture analysis from whole-body diffusion-weighted MRI. J Magn Reson Imaging. 2018. Oct;48(4):897-906.
14. Hedayati V, Tunariu N, Collins D, Koh D-M. Diffusion-Weighted MR Imaging in Oncology. Current Radiology Reports. 2014;2(5).

15. Giannarini G, Petralia G, Thoeny HC. Potential and limitations of diffusionweighted magnetic resonance imaging in kidney, prostate, and bladder cancer including pelvic lymph node staging: a critical analysis of the literature. Eur Urol. 2012;61(2):326-40.

16. Kwee TC, Takahara T, Luijten PR, Nievelstein RA. ADC measurements of lymph nodes: inter- and intra-observer reproducibility study and an overview of the literature. Eur J Radiol. 2010;75(2):215-20.

17. Freihat $O$, Pinter T, Kedves A, Sipos D, Cselik Z, Repa I, et al. Diffusionweighted imaging (DWI) derived from PET/MRI for lymph node assessment in patients with head and neck squamous cell carcinoma (HNSCC). Cancer Imaging. 2020;20(1):56.

18. Song $Q, Y u Y$, Zhang $X$, Zhu Y, Luo Y, Yu T, et al. Value of MRI and diffusion weighted imaging in diagnosing normal-sized pelvic lymph nodes metastases in patients with cervical cancer. Br J Radiol. 2020;20200203.

19. Vezhnevets VK, V. "Grow-Cut" - Interactive Multi-Label N-D Image Segmentation". Proc. Graphicon; 2005. p. 150-156.

20. Gray H. Anatomy of the human body. 20 ed. Lewis. WH, editor. Philadelphia: Lea \& Febiger, 1918; 2000. 692 p.

21. Som PM, Curtin HD, Mancuso AA. Imaging-based nodal classification for evaluation of neck metastatic adenopathy. AJR Am J Roentgenol. 2000; 174(3):837-44.

22. Park YM, Park JS, Yoon HK, Yang WT. Imaging-pathologic correlation of diseases in the axilla. AJR Am J Roentgenol. 2013;200(2):W130-42.

23. Walker CM, Chung JH, Abbott GF, Little BP, El-Sherief AH, Shepard JA, et al. Mediastinal lymph node staging: from noninvasive to surgical. AJR Am J Roentgenol. 2012;199(1):W54-64

24. Silverman B. Density estimation for statistics and data analysis. London: Chapman \& Hall/CRC; 1998

25. Moreau B, lannessi A, Hoog C, Beaumont H. How reliable are ADC measurements? A phantom and clinical study of cervical lymph nodes. Eur Radiol. 2018. Aug;28(8):3362-3371.

26. Ciritsis A, Rossi C, Marcon M, Van VDP, Boss A. Accelerated diffusionweighted imaging for lymph node assessment in the pelvis applying simultaneous multislice acquisition: a healthy volunteer study. Medicine (Baltimore). 2018;97(32):e11745.

27. Messiou C, Hillengass J, Delorme S, Lecouvet FE, Moulopoulos LA, Collins DJ, et al. Guidelines for acquisition, interpretation, and reporting of wholebody MRI in myeloma: myeloma response assessment and diagnosis system (MY-RADS). Radiology. 2019;291(1):5-13.

28. Padhani AR, Lecouvet FE, Tunariu N, Koh DM, De Keyzer F, Collins DJ, et al. METastasis reporting and data system for prostate Cancer: practical guidelines for acquisition, interpretation, and reporting of whole-body magnetic resonance imaging-based evaluations of multiorgan involvement in advanced prostate Cancer. Eur Urol. 2017;71(1):81-92.

29. Li S, Xue HD, Li J, Sun F, Jiang B, Liu D, et al. Application of whole body diffusion weighted MR imaging for diagnosis and staging of malignant lymphoma. Chin Med Sci J. 2008;23(3):138-44.

30. Koh DM, Blackledge M, Collins DJ, Padhani AR, Wallace T, Wilton B, et al. Reproducibility and changes in the apparent diffusion coefficients of solid tumours treated with combretastatin A4 phosphate and bevacizumab in a two-Centre phase I clinical trial. Eur Radiol. 2009;19(11):2728-38.

31. Just N. Improving tumour heterogeneity MRI assessment with histograms. Br J Cancer. 2014;111(12):2205-13.

32. De Paepe K, De Keyzer F, Wolter P, Bechter O, Janssens A, Dierickx D, et al. Whole-body diffusion-weighted imaging for staging lymphoma: are apparent diffusion coefficient derived histogram parameters useful for lesion characterisation? Cancer Imaging. 2014;14(Suppl 1):S9.

33. King AD, Chow KK, Yu KH, Mo FK, Yeung DK, Yuan J, et al. Head and neck squamous cell carcinoma: diagnostic performance of diffusion-weighted MR imaging for the prediction of treatment response. Radiology. 2013;266(2): 531-8.

34. Downey K, Riches SF, Morgan VA, Giles SL, Attygalle AD, Ind TE, et al. Relationship between imaging biomarkers of stage I cervical cancer and poor-prognosis histologic features: quantitative histogram analysis of diffusion-weighted MR images. AJR Am J Roentgenol. 2013;200(2):314-20.

35. Kyriazi S, Collins DJ, Messiou C, Pennert K, Davidson RL, Giles SL, et al. Metastatic ovarian and primary peritoneal cancer: assessing chemotherapy response with diffusion-weighted MR imaging--value of histogram analysis of apparent diffusion coefficients. Radiology. 2011;261(1):182-92. 
36. Wang F, Wang Y, Zhou Y, Liu C, Liang D, Xie L, et al. Apparent diffusion coefficient histogram analysis for assessing tumor staging and detection of lymph node Metastasis in epithelial ovarian Cancer: correlation with p53 and Ki-67 expression. Mol Imaging Biol. 2019;21(4):731-9.

37. Blackledge MD, Tunariu N, Orton MR, Padhani AR, Collins DJ, Leach MO, et al. Inter- and intra-observer repeatability of quantitative whole-body, diffusion-weighted imaging (WBDWI) in metastatic bone disease. PLoS One. 2016;11(4):e0153840.

38. Crescenzi R, Donahue PM, Braxton VG, Scott AO, Mahany HB, Lants SK, et al. 3.0 T relaxation time measurements of human lymph nodes in adults with and without lymphatic insufficiency: Implications for magnetic resonance lymphatic imaging. NMR Biomed. 2018;31(12):e4009.

39. Malyarenko DI, Ross BD, Chenevert TL. Analysis and correction of gradient nonlinearity bias in apparent diffusion coefficient measurements. Magn Reson Med. 2014 Mar:71(3):1312-23.

\section{Publisher's Note}

Springer Nature remains neutral with regard to jurisdictional claims in published maps and institutional affiliations.

Ready to submit your research? Choose BMC and benefit from:

- fast, convenient online submission

- thorough peer review by experienced researchers in your field

- rapid publication on acceptance

- support for research data, including large and complex data types

- gold Open Access which fosters wider collaboration and increased citations

- maximum visibility for your research: over $100 \mathrm{M}$ website views per year

At BMC, research is always in progress.

Learn more biomedcentral.com/submissions 